\title{
The Database for Inventory, Monitoring, and Assessment (DIMA)
}

\section{By Ericha M. Courtright and Justin W. Van Zee}

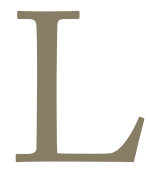

and managers increasingly need to collect, store, and analyze monitoring and assessment data sets that include multiple methods and occur over multiple years. For this reason, databases are becoming increasingly important for managing large monitoring and assessment data sets. The Database for Inventory, Monitoring, and Assessment (DIMA) is a highly customizable software tool for data collection, management, and interpretation. DIMA is a free Microsoft Access database that can be used easily without extensive knowledge of Access. ${ }^{i}$ All that is needed to run DIMA is a PC computer with a copy of Microsoft Access. Data can be entered for common, nationally accepted, vegetation- and soilmonitoring methods in the field using a tablet PC (touchscreen entry) or in the office on a standard computer (keyboard entry) with user-specified choice lists. DIMA can easily be customized to suit the user's needs.

DIMA was originally designed as an accompaniment to the Monitoring Manual for Grassland, Shrubland, and Savanna Ecosystems $^{1,2}$ (i.e., the Monitoring Manual). Just as the Monitoring Manual outlined a consistent approach to many field methods, DIMA provides a consistent approach to the data collection of those methods. Initially, DIMA supported only a few core vegetation- and soil-monitoring methods, but it has since grown to hold the quantitative data collected according to the standard methods outlined in the Monitoring Manual, the qualitative data collected according to Interpreting Indicators of Rangeland Health, ${ }^{3}$ and data collected using other nationally recognized methods (Table 1). Finally, data for developing Natural Resources Conservation Service (NRCS) ecological site descriptions can be collected using DIMA starting at a low intensity (e.g., general site characteristics, waypoints, and photos) and building to a high intensity (e.g., detailed soil and vegetation

'DIMA can be downloaded from http://jornada.nmsu.edu/monit-assess/ dima/download. data). In this article, we explain how DIMA facilitates the collection, management, and interpretation of field data (Fig. 1), helping users make informed decisions, think about data in new ways, and link that data to other sources.

\section{Preparation and Set Up}

Prior to performing fieldwork, DIMA is prepared in the office with the following information: personnel, including contacts and field crew members; county list for each state; state plant species lists downloaded from PLANTS database ${ }^{4}$; community species lists in which known plants for a particular area are identified; driving directions to the site if necessary; method rule sets; soils data; links to photos; general site and plot details; and other metadata as required or needed by the user.

The use of standard plant codes in DIMA facilitates consistency in the collection and storage of data and makes collection of data in the field faster. Plant species codes for a state can be downloaded from the online PLANTS database. Customized plant species codes can also be imported from Excel. This is useful when working on historic projects or for projects outside the United States. Each plant species code can be identified by growth form (e.g., tree, shrub, sub-shrub, succulent, forb/herb, graminoid) and duration (e.g., annual, perennial, biennial). Unknown plant codes can be created in the field when unidentifiable plants are encountered. These plant codes are easily replaced using a built-in tool that finds the temporary, unknown code and replaces it with the correct code.

Often DIMA needs to be prepared for a field crew who will collect a particular set of data. Several steps can be taken beforehand to eliminate errors in the field and to speed up data collection. First, the methods in the data entry screen can be edited to display only those required for a project. Second, a community species list can be created containing the plants expected to occur on the field plots. A community species list reduces the possibility of data entry errors and speeds up data entry by providing a simplified list of the 
Table 1. Methods contained in the Database for Inventory, Monitoring, and Assessment

\begin{tabular}{|l|}
\hline Vegetation \\
\hline Dry weight rank \\
\hline Gap intercept \\
\hline Canopy gap w/species $^{*}$ \\
\hline Line-point intercept \\
\hline Continuous line intercept \\
\hline Nested frequency \\
\hline Ocular estimates \\
\hline Plant density/belt transect \\
\hline Plant production* \\
\hline Species richness \\
\hline Tree inventory (includes density)* \\
\hline Vegetation structure (cover pole/board)* \\
\hline Riparian \\
\hline
\end{tabular}

\section{Channel/gully profile*}

\begin{tabular}{|c|}
\hline Soils \\
\hline Infiltration* \\
\hline Soil compaction (impact penetrometer)* \\
\hline Soil stability* \\
\hline Qualitative assessment \\
\hline Rangeland health \\
\hline Ecological inventory data \\
\hline $\begin{array}{l}\text { National Resources Conservation Service (NRCS) eco- } \\
\text { logical site description data can be collected starting at a } \\
\text { low intensity (e.g., general site characteristics, waypoints, } \\
\text { and photos) and building to a high intensity (e.g., detailed } \\
\text { soil and vegetation data) }\end{array}$ \\
\hline $\begin{array}{l}\text { *Methods described in the Monitoring Manual for Grassland, } \\
\text { Shrubland, and Savanna Ecosystems, }{ }^{1,2} \text { which has been } \\
\text { formally endorsed for rangeland monitoring by the Bureau } \\
\text { of Land Management and NRCS. }\end{array}$ \\
\hline
\end{tabular}

plant species typically found in the area. Third, the default rule set for each method can be defined in advance. This ensures, for example, that each transect of line-point intercept data will be collected from a $50-\mathrm{m}$ line at $100-\mathrm{cm}$ intervals with an ad hoc height option and a checkbox option for dead plants. Finally, details like crew-member names can be entered in the desired format (e.g., initials or full names). In as little as 30 minutes, DIMA can be set up with the site, plot, and transect details including global positioning system (GPS) coordinates, as well as a plant species list for the state, an abbreviated plant species list for the plots, data methods, and rule sets for those methods.
In the office, DIMA can also be set up with site and plot details, as well as field methods and customized defaults for those methods. In DIMA, a site is defined as a geographic or management area (e.g., ranch, military base, or conservation area). Each site may include multiple plots, and each plot contains one or more transects. Figure 2 illustrates the relationship between sites, plots, and transects.

Sometimes a user wants to use DIMA without any preparation in the office. This is a common occurrence when working on ecological site descriptions. Within the low-intensity ecological inventory method, a site and/or plot can be created on the fly, and information including plot characteristics, waypoints, and waypoint photos can be entered. In addition, very specialized data for ecological site descriptions or projects that require more detailed information may be entered into data sets for the medium- and high-intensity ecological inventory method. This method includes very detailed soil pits, pedon attributes, plant composition, soil-surface attributes, soil taxons, ecological site/states, and specialized plot information.

\section{Data Collection}

After DIMA is set up in the office, it can be loaded onto a tablet PC that is used for field data collection (Fig. 3). DIMA is easy to use in the field, with its drop down menus, quick data entry forms, lists that are sorted by frequency of use (e.g., the most common plant species codes automatically move to the top of the list for line-point intercept), and an auto-save feature that is triggered each time a data collection screen is closed (Fig. 4). Utilizing DIMA on a tablet PC to collect data electronically in the field eliminates countless hours of data entry and error checking. DIMA reinforces consistent data collection through the use of plant species codes, collection of basic metadata, nationally recognized soil and vegetation field methods, and warning messages when there is a problem with the data (e.g., missing or mismatched data).

DIMA also has the capability of capturing location information directly from a GPS unit attached to the field computer. An external GPS unit can be attached to the field computer via a USB or serial COM port, or a built-in GPS unit can be used to collect GPS coordinates directly in DIMA. By collecting the GPS coordinates directly, data entry and error checking of the coordinates are eliminated. Additionally, if mapping software such as the Environmental Science Research Institute's ArcPad or Google Earth is installed on the field computer, the attached GPS unit can also be used to navigate to the plot, especially if the location has been preselected.

In some cases, multiple tablet PCs are used for data collection, and each computer will have a copy of DIMA with only a portion of the total field data. At the end of the field campaign, these databases are easily merged into one by importing the data from each field copy of DIMA 


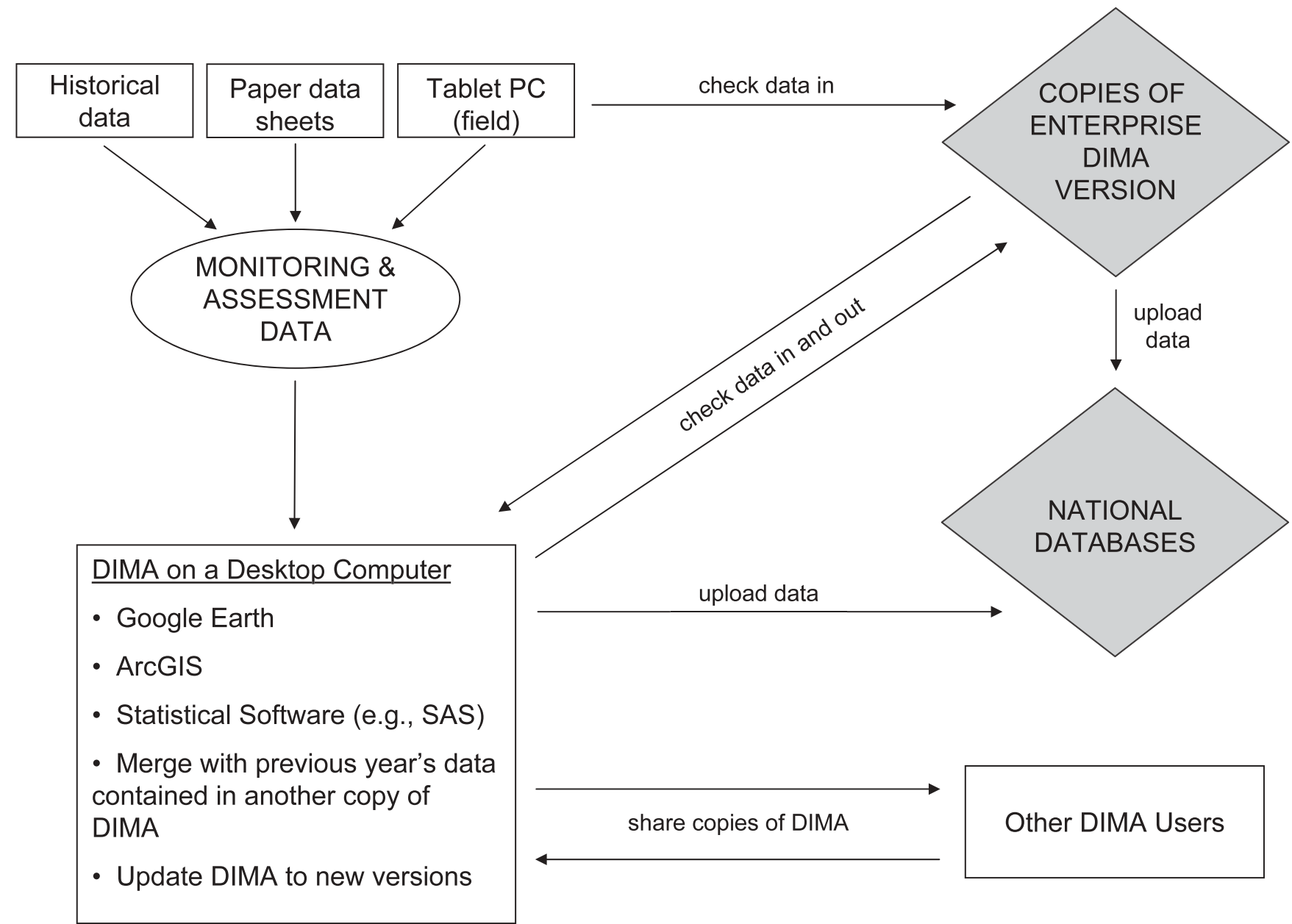

Figure 1. Diagram outlining the flow of data through the Database for Inventory, Monitoring, and Assessment (DIMA) and how DIMA interacts with other software and databases. Shaded objects represent future enhancements.

into a single, master copy of DIMA. This procedure takes $<5$ minutes and results in a comprehensive data set.

Data collected during previous field seasons on paper data sheets and entered and stored in Excel can be imported into the database using import functions programmed for each data method. This procedure is a little more time consuming because the Excel spreadsheets have to be manipulated to match the import program. Once the Excel spreadsheets match the import template, all data can be imported and stored in DIMA. Importing the data is much quicker than re-entering all of the data manually into the database and checking for errors (i.e., requires hours vs. weeks).

\section{Data Manipulation}

Data manipulation is the calculation of indicators from the raw data; indicators are calculated for transect, plot, and site. These indicators can then be used in reports or in other statistical packages for additional data analyses. For example, DIMA calculates the amount of foliar cover from the line-point intercept method. Indicator calculations are automatically generated for the field methods. Currently, simple calculations on the data entry forms within DIMA exist for all the methods. DIMA calculates averages instantly for each method and displays them on the same screen used for the data entry. By examining this information, outliers or data entry errors from fieldwork can be detected in the field before leaving the plot.

More complete indicator calculations and descriptive statistics are available as reports for line-point intercept, gap intercept, plant density, and method tracking. These data summaries can be all-inclusive over many years or can be broken down by year for selected indicators (e.g., plant growth form, percentage of bare soil, basal cover, lichen crust, etc.). A commonly run report is the indicators report for line-point intercept data. This report can be run on data for a single year or for multiple years. When multiple years of data are selected and "Show Totals by Year" is checked, the indicators are calculated for each year. The user chooses which indicators to include in the report. There are currently 33 indicators from which to choose, including percentage of bare soil, percentage of total foliar cover, and percentage of cover of each plant species. Indicators are calculated for each transect, for the transects averaged for 


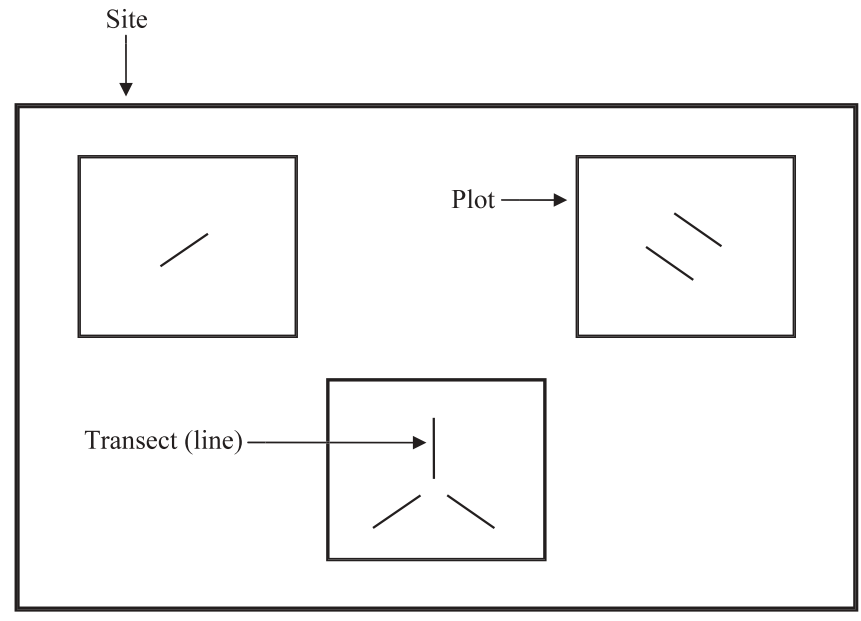

Figure 2. Relationship between sites, plots, and transects. Each plot can have one or more transects, and each site can have one or more plots. Borders around the site and plots are for illustration purposes only and have no meaning in the field.

each plot, and for the plots averaged for each site. For indicators that occur in the upper and lower layers of line-point intercept, the "top hit" and "any hit" are calculated. Standard deviations are calculated for plot and site averages. These

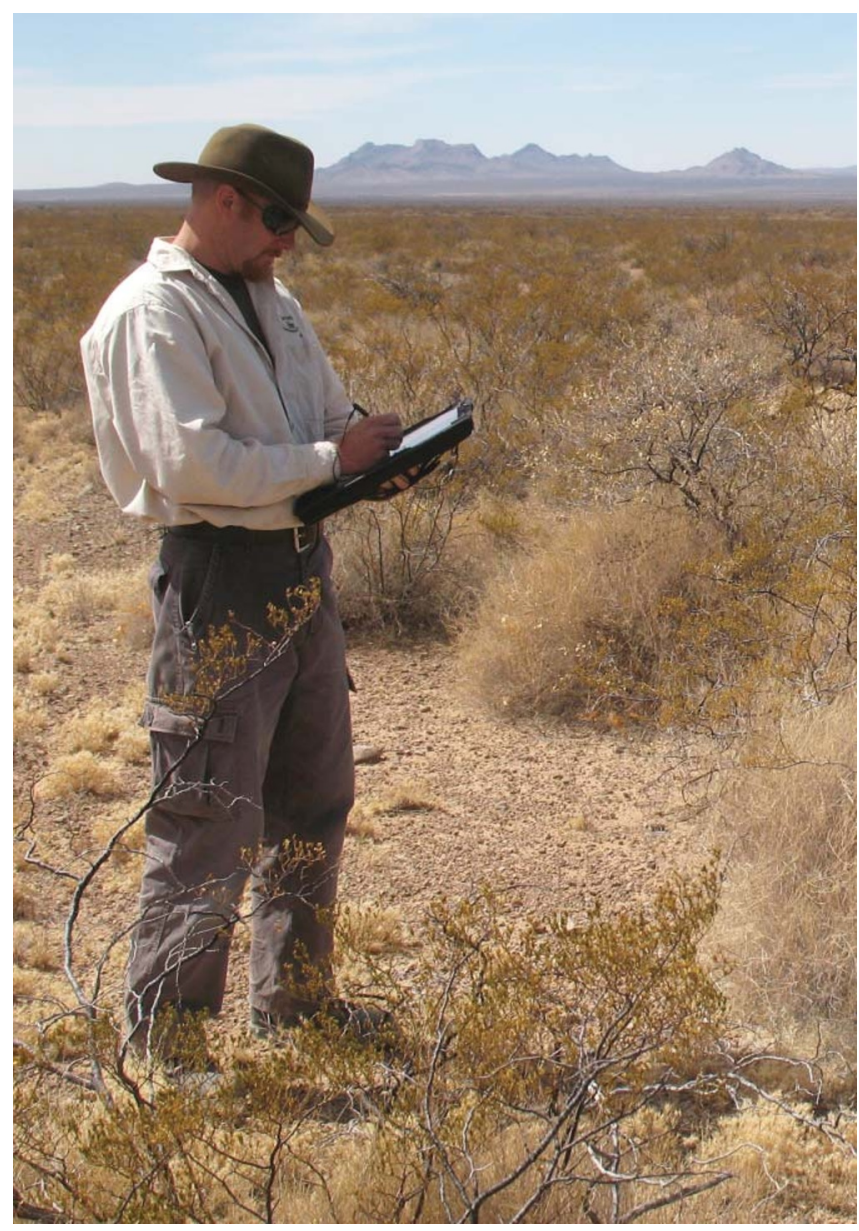

Figure 3. The portability of tablet PCs makes the Database for Inventory, Monitoring, and Assessment extremely valuable even at remote field sites.

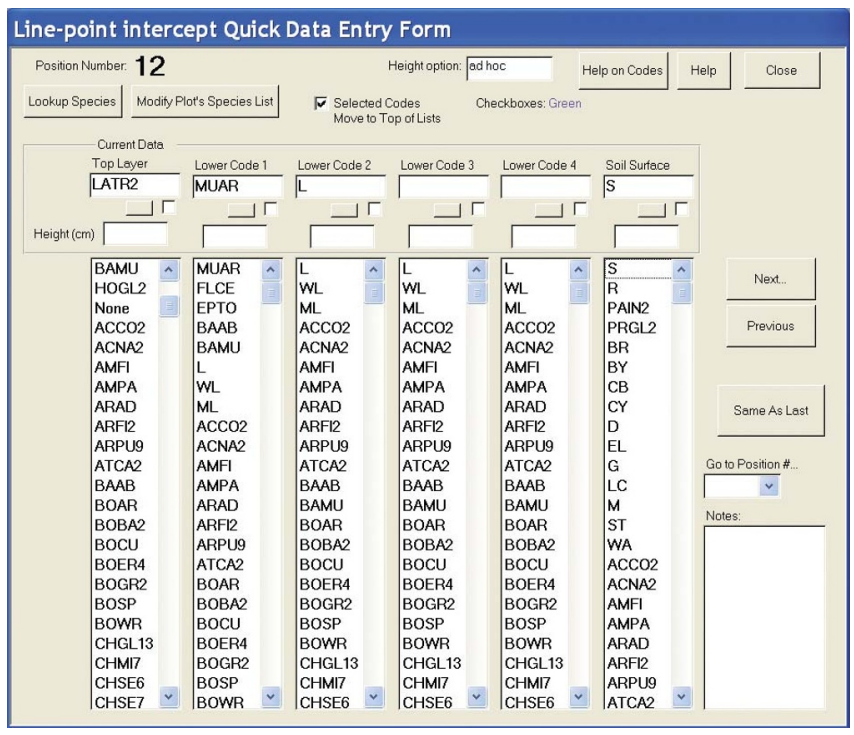

Figure 4. Screen shot of the line-point intercept quick data entry form in the Database for Inventory, Monitoring, and Assessment. Note that the plant codes are ordered with the most commonly used codes at the top of the list.

reports are output to Excel, so the user can produce tables for a project report or perform additional analyses with other software (Fig. 5). In addition to the reports, anything from the entire database to small portions of the database can be exported into an Excel file or exported as a delimited text file based on date, site, or field method. This option allows the user to export data and run analyses with other statistical software (e.g., SAS).

When working with historical data, users frequently wish to identify and categorize plots into various subgroups that may differ from the initial study. Sites help identify plots, but they are limited because a plot can only be relegated to a single site, and a site is typically a geographic delineation. For instance, a plot can be identified in many different ways depending on the specific elements of the data analysis. Sometimes a control plot can also be identified by an ecotype (e.g., shrub steppe) and characterized as ideal for certain wildlife (e.g., sage grouse). These identifiers are important in terms of how data is considered and analyzed. Plot tags were created to facilitate this type of identification. Plot tags allow the user to categorize the data in an unlimited fashion by labeling a plot with customized key identifiers (e.g., control, vehicle disturbance, roads, restoration, research, etc.) in addition to the site and plot names. Reports can be run based on the plot tags, thereby allowing the user to compare a control area to a disturbed or treatment area.

\section{Advantages and Limitations}

DIMA allows data to be contained and stored in one location, easily shared with others, and merged with other sets of data stored in separate copies of DIMA. In addition to storing field data, important metadata such as field notes, GPS coordinates, collection dates, and collection personnel 


\begin{tabular}{|c|c|c|c|c|c|c|c|c|c|c|c|c|c|c|c|c|c|c|}
\hline & A & $B$ & C & D & $E$ & $F$ & G & $\mathrm{H}$ & 1 & & K & L & M & $\mathrm{N}$ & 0 & $\mathrm{P}$ & 0 & $R$ \\
\hline 1 & & & & & 2006 & & & & & 2007 & & & & & 2008 & & & \\
\hline 2 & Site & Plot L & Line & Indicator & & 1st Hit Avg & Any Hit N $F$ & Any Hit Avg & & & 1st Hit Avg & Any Hit $\mathrm{N}$ & Any Hit Avg & & & 1st Hit Avg & Any Hit N & Any Hit Avg \\
\hline 3 & CBR & 1 & & Bare Soil & 11 & 0.220 & & & & 10 & 0.200 & & & & 38 & 0.760 & & \\
\hline 4 & CBR & 1 & & Litter & 25 & 0.500 & 0 & 0.000 & & 18 & 0.360 & 0 & 0.000 & & 7 & 0.140 & 0 & 0.000 \\
\hline 5 & CBR & 1 & & ARPU & 1 & 0.020 & 1 & 0.020 & & 1 & 0.020 & 1 & 0.020 & & 0 & 0.000 & & 0.000 \\
\hline 6 & CBR & 1 & & BOER & 9 & 0.180 & 10 & 0.200 & & 6 & 0.120 & 8 & 0.160 & & 0 & 0.000 & & 0.000 \\
\hline 7 & CBR & 1 & & BOGR & 7 & 0.140 & 8 & 0.160 & & 17 & 0.340 & 18 & 0.360 & & 0 & 0.000 & 0 & 0.000 \\
\hline 8 & CBR & 1 & & PG & 0 & 0.000 & 0 & 0.000 & & 0 & 0.000 & 0 & 0.000 & & 3 & 0.060 & 3 & 0.060 \\
\hline 9 & CBR & 1 & & SPHA & 0 & 0.000 & 0 & 0.000 & & 1 & 0.020 & 1 & 0.020 & & 0 & 0.000 & 0 & 0.000 \\
\hline 10 & CBR & 1 & & STNE & 9 & 0.180 & 9 & 0.180 & & 7 & 0.140 & 8 & 0.160 & & 0 & 0.000 & 0 & 0.000 \\
\hline 11 & CBR & 1 & & THAC & 3 & 0.060 & 3 & 0.060 & & 1 & 0.020 & 1 & 0.020 & & 1 & 0.020 & 1 & 0.020 \\
\hline 12 & CBR & 1 & & ZIGR & 0 & 0.000 & 0 & 0.000 & & 2 & 0.040 & 2 & 0.040 & & 0 & 0.000 & 0 & 0.000 \\
\hline 13 & CBR & 1 & & Total Folliar & 29 & 0.580 & 29 & 0.580 & & 35 & 0.700 & 35 & 0.700 & & 4 & 0.080 & 4 & 0.080 \\
\hline 14 & & & & & & & & & & & & & & & & & & \\
\hline 15 & CBR & 1 & 120 & Bare Soil & 15 & 0.300 & & & & 8 & 0.160 & & & & 29 & 0.580 & & \\
\hline 16 & CBR & 1 & 120 & Litter & 21 & 0.420 & 0 & 0.000 & & 23 & 0.460 & 0 & 0.000 & & 10 & 0.200 & 0 & 0.000 \\
\hline 17 & CBR & 1 & 120 & BOER & 2 & 0.040 & 2 & 0.040 & & 5 & 0.100 & 7 & 0.140 & & 0 & 0.000 & 0 & 0.000 \\
\hline 18 & CBR & 1 & & BOGR & 17 & 0.340 & 17 & 0.340 & & 20 & 0.400 & 22 & 0.440 & & 0 & 0.000 & & 0.000 \\
\hline 19 & CBR & & 120 & CYIM & 0 & 0.000 & 0 & 0.000 & & 1 & 0.020 & 1 & 0.020 & & 0 & 0.000 & & 0.000 \\
\hline 20 & CBR & 1 & 120 & GUSA & 1 & 0.020 & 1 & 0.020 & & 2 & 0.040 & 2 & 0.040 & & 0 & 0.000 & & 0.000 \\
\hline 21 & CBR & $i$ & 120 & MUTO & 0 & 0.000 & 0 & 0.000 & & 0 & 0.000 & 1 & 0.020 & & 0 & 0.000 & 0 & 0.000 \\
\hline 22 & CBR & 1 & 120 & PAOB & 0 & 0.000 & 0 & 0.000 & & 0 & 0.000 & 1 & 0.020 & & 0 & 0.000 & 0 & 0.000 \\
\hline 23 & CBR & 1 & 120 & & 0 & 0.000 & 0 & 0.000 & & 0 & 0.000 & 0 & 0.000 & & 10 & 0.200 & 10 & 0.200 \\
\hline 24 & CBR & 1 & 120 & SELE & 0 & 0.000 & 1 & 0.020 & & 0 & 0.000 & 0 & 0.000 & & 0 & 0.000 & 0 & 0.000 \\
\hline 25 & CBR & 1 & 120 & SOEL & 0 & 0.000 & 0 & 0.000 & & 0 & 0.000 & 1 & 0.020 & & 0 & 0.000 & 0 & 0.000 \\
\hline 26 & CBR & 1 & 120 & SPHA & 0 & 0.000 & 0 & 0.000 & & 2 & 0.040 & 4 & 0.080 & & 0 & 0.000 & 0 & 0.000 \\
\hline 27 & CBR & 1 & 120 & STNE & 1 & 0.020 & 2 & 0.040 & & 0 & 0.000 & 0 & 0.000 & & 0 & 0.000 & 0 & 0.000 \\
\hline 28 & CBR & 1 & 120 & YUEL & 2 & 0.040 & 2 & 0.040 & & 2 & 0.040 & 2 & 0.040 & & 0 & 0.000 & 0 & 0.000 \\
\hline 29 & CBR & 1 & 120 & Total Foliar & 23 & 0.460 & 23 & 0.460 & & 32 & 0.640 & 32 & 0.640 & & 10 & 0.200 & 10 & 0.200 \\
\hline 30 & & & & & & & & & & & & & & & & & & \\
\hline 31 & CBR & 1 & 240 & Bare Soil & 15 & 0.300 & & & & 9 & 0.180 & & & & 36 & 0.720 & & \\
\hline 32 & CBR & 1 & 240 & Litter & 19 & 0.380 & 0 & 0.000 & & 14 & 0.280 & 0 & 0.000 & & 9 & 0.180 & 0 & 0.000 \\
\hline 33 & CBR & 1 & 240 & BOER & 4 & 0.080 & 6 & 0.120 & & 11 & 0.220 & 12 & 0.240 & & 0 & 0.000 & 0 & 0.000 \\
\hline 34 & CBR & 1 & 240 & BOGR & 7 & 0.140 & 7 & 0.140 & & 15 & 0.300 & 17 & 0.340 & & 1 & 0.020 & 1 & 0.020 \\
\hline 35 & CBR & 1 & 240 & GUSA & 1 & 0.020 & 1 & 0.020 & & 2 & 0.040 & 2 & 0.040 & & 0 & 0.000 & 0 & 0.000 \\
\hline 36 & CBR & 1 & 240 & $P G$ & 0 & 0.000 & 0 & 0.000 & & 0 & 0.000 & 0 & 0.000 & & 3 & 0.060 & 3 & 0.060 \\
\hline 37 & CBR & 1 & 240 & SPFL & 0 & 0.000 & 0 & 0.000 & & 1 & 0.020 & 1 & 0.020 & & 0 & 0.000 & 0 & 0.000 \\
\hline 38 & CBR & 1 & 240 & STNE & 7 & 0.140 & 7 & 0.140 & & 5 & 0.100 & 5 & 0.100 & & 0 & 0.000 & 0 & 0.000 \\
\hline 39 & CBR & 1 & & Total Foliar & 19 & 0.380 & 19 & 0.380 & & 34 & 0.680 & 34 & 0.680 & & 4 & 0.080 & 4 & 0.080 \\
\hline
\end{tabular}

Figure 5. Excel output from the line-point-intercept indicators report. Three years of data are displayed for three transects on the same plot.

(recorder and observer) are stored. When new versions of DIMA are released, the user merely downloads the new version from the Internet ${ }^{\text {ii }}$ and then imports prior versions of DIMA data, so data is never lost or abandoned due to program updates.

Because DIMA is built in Access, users are not locked into using the predefined reports, and they are allowed to design their own queries. These queries can link any of the data collected or the data summaries together to answer larger questions that require combining different forms of data, like vegetation and soils. For example, soil-pit data and vegetation characteristics may be of interest. The ability to write queries adds another layer of flexibility to DIMA.

DIMA is currently used by a number of organizations worldwide for short- and long-term monitoring, and for collecting quantitative data to support rangeland assessments. It also facilitates qualitative and quantitative data collection to assist with developing soil- and vegetation-based landclassification systems (e.g., ecological site descriptions) and populates them with quantitative soil and vegetation data. Because the tool is extremely flexible, it can be adapted to meet the needs of a diverse group of users, from students compiling data for a course to scientists developing research projects to land management agencies responsible for longterm monitoring. Currently, DIMA is used by the USDA Agricultural Research Service, United States Geological Survey (USGS), NRCS, Bureau of Land Management (BLM), universities, private environmental consultants, and the Mongolian Society of Range Management. Examples of data projects stored within DIMA have ranged from

iUpdated versions of DIMA can be downloaded at http://jornada.nmsu. edu/monit-assess/dima/download. rangeland surveys and ecological sites in Mongolia, ecological site development by the National Resources Inventory, and rangeland monitoring on various Department of Defense properties, National Park Service lands, and BLM units. It is also used by private contractors.

As with all software, DIMA has its limitations as well as a few known issues. New versions of DIMA are released several times per year. New releases fix bugs discovered since the previous release and provide new functionality and features. Users need to keep DIMA updated in order to take advantage of these improvements. When working with multiple copies of DIMA in the field or on projects that cover multiple years, data need to be merged into a single copy of DIMA to prevent data loss. As with all data, metadata need to be entered in order to make the data meaningful. To prevent the loss of data if a hard drive fails, DIMA should be backed up regularly. In most cases, DIMA is not flexible enough to house experimental data for new field methods. As with all data, data entry errors may exist and need to be addressed. DIMA is not functional on Apple computers because a Mac version of Microsoft Access is not available.

\section{New and Future Enhancements}

DIMA has come a long way from its initial stage of collecting data for a few methods such as line-point intercept, gap intercept, and rangeland health. Currently work is ongoing to increase DIMA's geospatial capabilities by providing 1) the ability to display locations and data for plots and transects on a map such that the data can be used in conjunction with other data and 2) planning and analysis tools for creating sampling locations and analyzing data. This geospatial integration was introduced with the map creation in Google Earth and will continue with the linkage to ArcGIS.

In addition to field data, plot and transect coordinates can now be stored in DIMA. These plot coordinates can be 
mapped from DIMA in Google Earth, creating a KML file. These files will be helpful in the field, particularly for creating ecological site descriptions and identifying ecological site boundaries once additional information such as soil-pit data is linked to the plot coordinates. Currently a small amount of metadata is grouped with each point, including aspect, ecological site, landform, location, map unit, major land-resource area type, region, slope, and soil series. In future releases, soil data, as well as data summaries from line-point intercept and gap intercept, will be linked to each set of plot coordinates in Google Earth. These summaries will include percent bare ground and percent foliar cover as well as other indicators.

Soon DIMA will link to ArcGIS, allowing soil and vegetation data to be mapped for geospatial interpretations of ecosystems. Data from ArcGIS will also be uploadable to DIMA, allowing users to perform plot selection in ArcGIS and then load the new plot coordinates into DIMA.

Work has also begun on an enterprise version of DIMA. Whereas DIMA is currently a standalone database run in Access on a personal computer, the enterprise version will be housed on secure servers and capable of handling hundreds of thousands of data sets. The enterprise version will contain data from numerous projects within a state and/ or across states and will be implemented either within a land-management agency or across agencies. New data will be "checked in" to the enterprise version, while copies of existing data can be "checked out" for data analyses. As other agencies create specialized databases or national databases (e.g., the USGS Land Treatment Digital Library), DIMA can be set up to interface with those databases.

DIMA has evolved and continues to evolve with more methods, report indicators, and tools included in each new release. The ongoing development of DIMA has resulted from direct requests, feedback, and needs of the people using it. As a result, DIMA is constantly being made better and more flexible. In addition to being responsive to user needs, technical support is provided via phone and e-mail.
Additional support is provided on the Web site, including Webinars and a frequently-asked-questions page. iii Upcoming training materials and videos will walk the user through a step-by-step process for everything from setting up DIMA to how to run a report. Finally, individual Webinar training will be offered to agency field offices, providing a more customized approach to dealing with predetermined issues or to answer questions for that particular group of users.

\section{References}

1. Herrick, J. E., J. W. Van Zee, K. M. Havstad, L. M. Burkett, and W. G. Whitford. 2005. Monitoring manual for grassland, shrubland, and savanna ecosystems. Volume 1: quick start. USDA-ARS Jornada Experimental Range, Las Cruces, NM, USA: University of Arizona Press. 36 p.

2. Herrick, J. E., J. W. Van Zee, K. M. Havstad, L. M. Burkett, and W. G. Whitford. 2005. Monitoring manual for grassland, shrubland, and savanna ecosystems. Volume 2: design, supplementary methods and interpretation. USDA-ARS Jornada Experimental Range, Las Cruces, NM, USA: University of Arizona Press. 200 p.

3. Pellant, M., P. Shaver, D. A. Pyke, and J. E. Herrick. 2005. Interpreting indicators of rangeland health, version 4. Denver, CO, USA: US Dept of the Interior, Bureau of Land Management, National Science and Technology Center. Technical Reference 1734-6. 122 p.

4. USDA, NRCS. 2011. The PLANTS database. Baton Rouge, LA, USA: National Plant Data Center. Available at: http:// plants.usda.gov. Accessed 6 May 2011.

Authors are Science Specialist, ericha@nmsu.edu (Courtright), and Biological Science Soils Technician (Van Zee), US Dept of Agriculture-Agricultural Research Service, Jornada Experimental Range, Las Cruces, NM 88003-8003, USA.

\footnotetext{
iii For frequently asked questions about DIMA, see http://jornada.nmsu. edu/monit-assess/dima.
} 